\title{
LA ESTRUCTURA DE UN CURSO EN LÍNEA Y EL USO DE LAS DIMENSIONES DEL APRENDIZAJE COMO MODELO INSTRUCCIONAL
}

\author{
M.C. Lewis McAnally-Salas \\ M.C. Carolina Armijo de Vega \\ Universidad Autónoma de Baja California
}

\section{Introducción}

A la par del crecimiento de la Educación en línea han crecido las opciones para crear o diseñar cursos en línea; en el mercado actualmente se ofertan programas de autoría para "armar" cursos en línea (Front Page $\AA$, Dreamweaver $\AA$, Go Live $\AA$, Hot Dog $\AA$, etc), y sistemas integrados (Learning Space $\AA$, Virtual- $U \AA$, Blackboard $\AA$, WebCT $\AA$, etc) que proporcionan capacidades variables para la administración y seguimiento de los mismos. Un abanico de alternativas tan amplias suele causar confusión a la hora de definir una estrategia para iniciar un curso en línea. Es común que la primera aproximación de los docentes a la Educación en línea sea mediante el uso de programas de autoría, cuya función primordial es el diseño de sitios de Red, con objeto de facilitar el acceso y uso de información, y no la creación de un sitio para impartir cursos en línea, cuya función primordial es facilitar el aprendizaje. Es usual que la falta de experiencia en la creación de ambientes de aprendizaje se convierta en una limitante que dificulte esta tarea, de ahí la necesidad de contar con una propuesta que presente alternativas, no sólo de la operación sino también de la estructura conveniente para la efectividad de los cursos. Por otro lado, si bien es cierto que por lo general la estructura de los cursos en línea en los sistemas integrados está definida por la misma plataforma, su operación es una actividad que suele quedar como responsabilidad del docente.

La instrumentación exitosa de un curso en línea no depende únicamente de factores técnicos relacionados con el diseño operativo y/o estético, ya que involucra factores humanos y pedagógicos determinantes. Por esta razón, sin pretender ser exhaustivos, esta propuesta considera algunos de estos aspectos como el diseño del ambiente de aprendizaje, su estructura, las tareas rutinarias, y el modelo instruccional adoptado.

\section{Fundamentos del diseño}

Para el diseño de esta propuesta se utiliza el modelo propuesto por Mclsaac y Gunawardena (1996) que describe los factores que se deben considerar para la selección y uso de tecnologías en cursos diseñados para educación a distancia, el cual ya fue utilizado con éxito en cursos en línea a nivel licenciatura y maestría (McAnally-Salas y Pérez-Fragoso, 2000). Según este modelo, las seis características más importantes que se deben considerar para la educación en línea son: a) transmisión y acceso, b) control, c) interacción, d) características simbólicas del medio, e) la presencia social creada a través del medio, y f) la interfaz entre el usuario y la máquina.

Dichas características se integran en el diseño de la presente propuesta, considerando las partes fundamentales que componen un curso, su función en el desarrollo del mismo y el modelo alrededor del cual se estructura. 


\section{Su organización}

La organización del curso gira alrededor de secciones que dividen los contenidos y actividades con base en su función principal (figura 1).

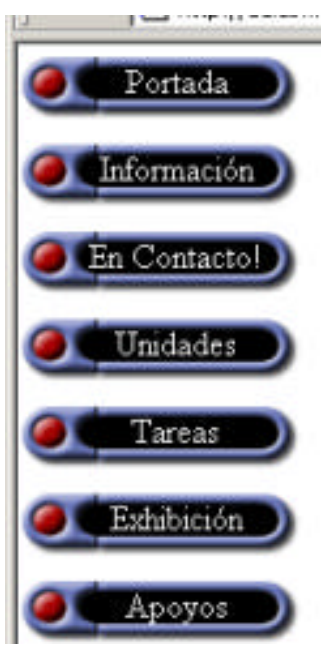

Figura 1. Menú que siempre está presente y que da acceso a las secciones principales del curso.

Aunque la portada no es una sección por sí misma, tiene una función primordial, ya que además de dar acceso a las secciones principales, mantiene al tanto a los estudiantes sobre el progreso del curso notificando las actividades de la semana y los avisos de ocasión pertinentes (figura 2).

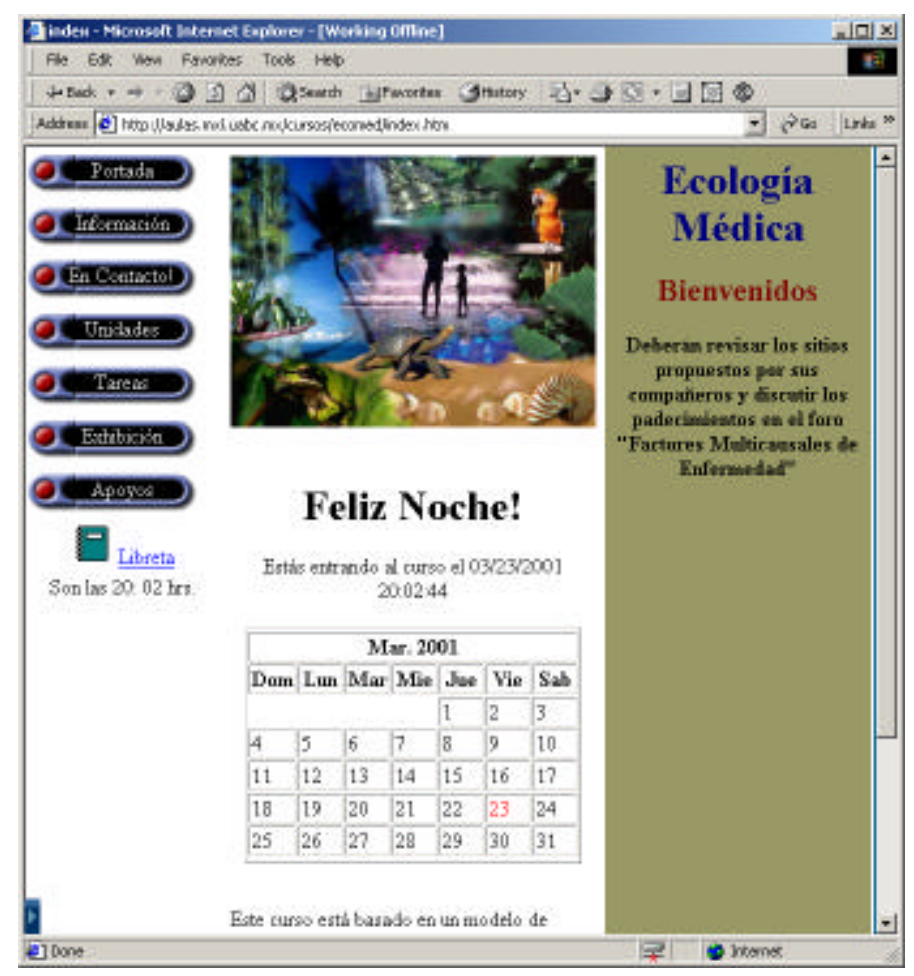

Figura 2. Portada o página de inicio de un curso en línea.

El nombre y características de las secciones principales son:

Información general, (figura 3) donde el objetivo es ofrecer al estudiante acceso a toda la información referente a las características del curso. 


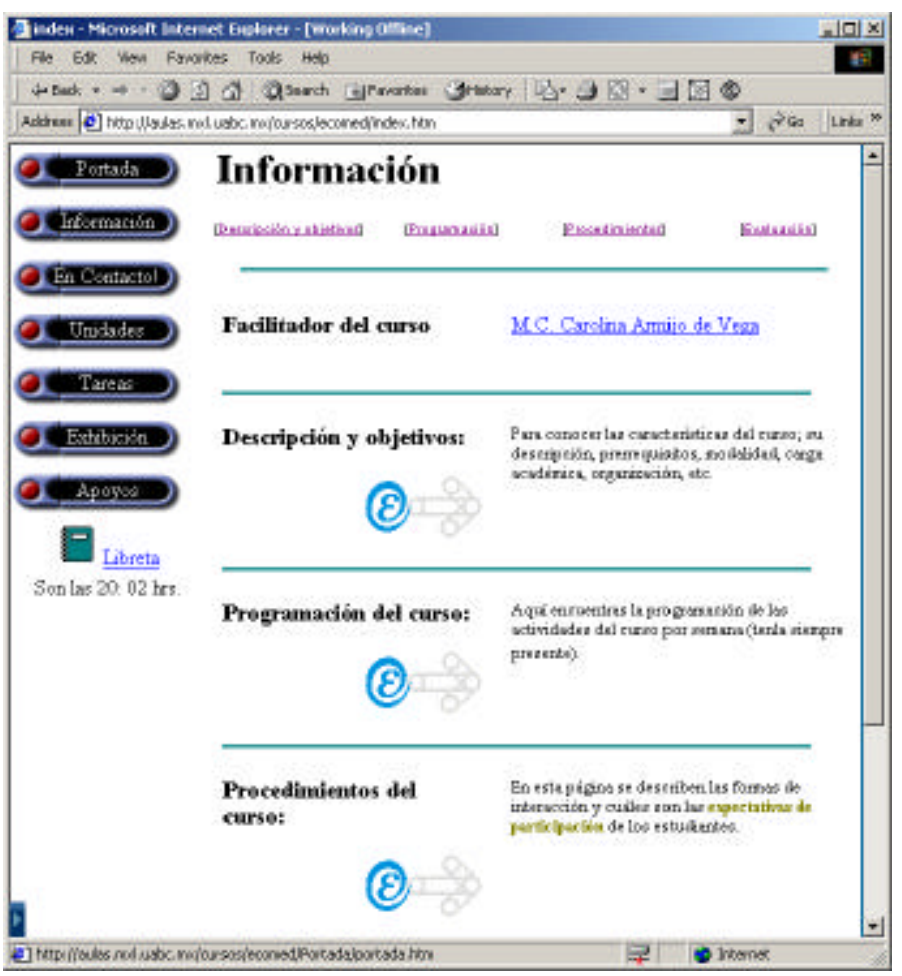

Figura 3. Sección de Información general en donde se muestran los hiperligas que llevan a la descripción detallada de cada parte del curso.

¡En Contacto!, (figura 4) es la sección de comunicación en la cual gira el desarrollo del curso, por lo que se integran cuatro módulos para la comunicación entre los participantes; Correos electrónicos de los participantes, dos organizadores de discusión asincrónica para los Foros de discusión (figura 5) y Charlas de café, y un programa de Charla en tiempo real o sincrónico.

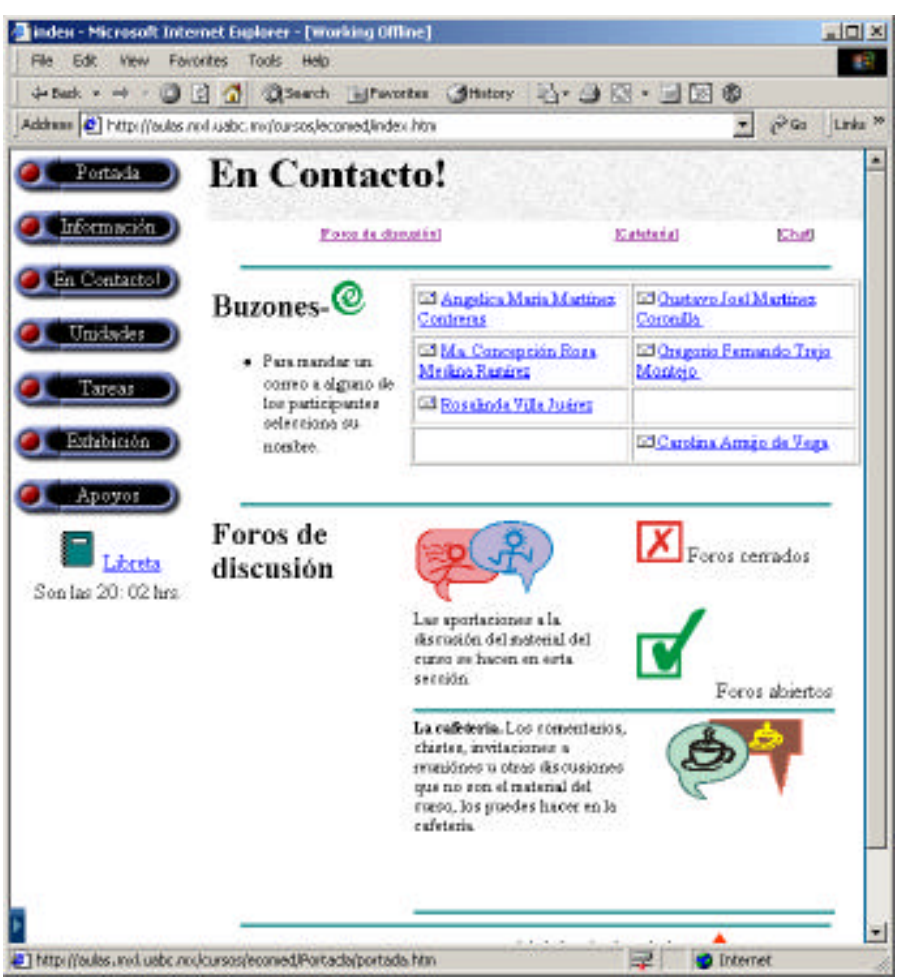

Figura 4. Sección de En Contacto en donde se muestra los hiperligas al correo electrónico de los participantes, a los foros de discusión y a la cafetería. 


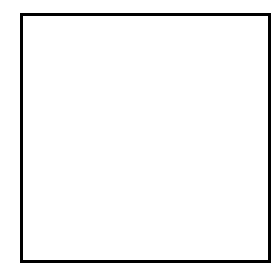

Figura 5. Foro de discusión que muestra la forma como se organizan las aportaciones de los participantes alrededor de un tema definido.

Unidades, (figura 6) proporciona acceso al temario, contenidos y tareas de cada una de las unidades que comprenden el curso.

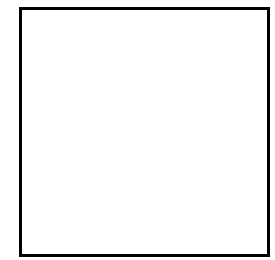

Figura 6. Página que muestra la sección de las unidades en donde se organizan los contenidos del curso.

Tareas, (figura 7) donde se describen las tareas por unidad y si es pertinente, se presentan hiperligas a las secciones relacionadas.

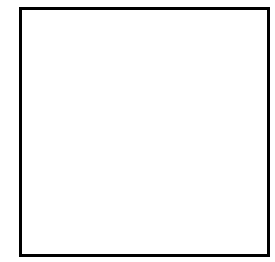

Figura 7. En la sección de tareas se organizan las actividades y se describen los productos esperados para cada unidad.

Exhibición, (figura 8) es la sección donde se publican todos los productos de los estudiantes, organizados por unidades.

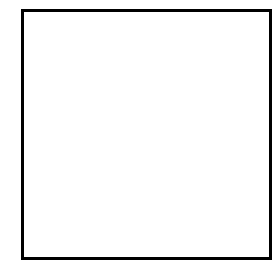

Figura 8. Una sección importante es la de exhibición en la cual se muestran los productos entregados por los participantes del curso.

Apoyo, donde se encuentra el acceso a tutoriales sobre el uso de algún programa a utilizar durante el curso (v. gr. el uso del programa de charla en tiempo real) o el procedimiento para realizar alguna acción (v. gr. instalar o configurar algún programa). En esta sección también se encuentran las hiperligas a sitios de interés relacionados con el curso.En esta ventana se encuentra también el acceso a la libreta de notas, pequeño programa para ir llevando sus propias notas del curso. 


\section{Estructura y navegación del curso}

Para facilitar la navegación dentro del curso, en algunos puntos del mismo al seleccionar la hiperliga que lleva a algún contenido, este se despliega en su propia ventana, es decir, el navegador se abre una vez más automáticamente con la información seleccionada. Las páginas que se despliegan en su propia ventana son las lecturas, foros de discusión, charla en tiempo real, etc., las cuales ya no tienen hiperligas al curso por lo que al terminar la actividad simplemente se cierra el navegador que se abrió automáticamente. El acceso a cualquier información del curso está a no más de tres clics de distancia.

Los espacios para las actividades no forzosamente se deben accionar desde la ventana principal, sino desde donde sea pertinente, así, la entrada a los Foros de discusión no será únicamente a través de la sección de En Contacto!, sino que puede ser a través de las Tareas de alguna Unidad.

\section{Modelo instruccional}

El modelo instruccional seleccionado es el de Marzano (1992), denominado Dimensiones del aprendizaje. Este modelo está basado en la premisa de que se requieren cinco tipos de pensamiento que son esenciales para el proceso de aprendizaje y que pueden ser apoyados con relativa facilidad por la modalidad en línea. A continuación se presenta una pequeña descripción de las dimensiones y ejemplos de aplicación para cursos en línea:

Dimensión 1: Actitudes y percepciones positivas acerca del aprendizaje, se refiere al hecho de que sin actitudes y percepciones positivas, los estudiantes difícilmente podrán aprender adecuadamente, de ahí que un elemento fundamental de la instrucción efectiva es lograr lo que busca esta dimensión.

Ejemplo: Al inicio de una unidad o tema enfrentar a los estudiantes ante una situación problematizadora que les permita tomar conciencia y motivarse positivamente hacia el contenido que será cubierto en esa parte del curso; esta actividad problematizadora puede ser una lista de conceptos que se tocarán en el curso y se solicita a los estudiantes que traten de definirlos y/o relacionarlos o bien una serie de datos impactantes relacionados con la temática del curso (v. gr. En un tema sobre contaminación de suelos pudiera ser el número de litros de aceite de motor que se tiran directamente al suelo por minuto, hora o día en la ciudad, estado o país). Esta actividad puede ser distribuida por correo-e o bien ser parte de la información en la página introductoria a la unidad o tema. Los comentarios relacionados pueden ser parte de la discusión en la cafetería virtual.

Dimensión 2: Adquisición e integración del conocimiento, se refiera a ayudar a los estudiantes a integrar el conocimiento nuevo con el conocimiento que ya se tiene, de ahí que las estrategias instruccionales para esta dimensión están orientadas a ayudar a los estudiantes a relacionar el conocimiento nuevo con el previo, organizar el conocimiento nuevo de manera significativa, y hacerlo parte de su memoria de largo plazo.

Ejemplo: En un curso de historia, solicitar a los estudiantes que lean (del material en línea o del libro de texto) los hechos relacionados con un episodio histórico utilizando la lista de discusión del curso y comentar lo que ya sabían del tema, qué eventos que conocen son consecuencia del episodio en cuestión, qué eventos en el presente son similares y qué pudiéramos predecir hacia el futuro; además, solicitar como tarea organizar la información en un diagrama que permita ver las relaciones 
alrededor del episodio y enviarlo por attachment para ser analizado y publicado en la sección de exhibición del curso.

Dimensión 3: Extender y refinar el conocimiento, se refiere a que el educando añade nuevas distinciones y hace nuevas conexiones. Analiza lo que ha aprendido con mayor profundidad y mayor rigor. Las actividades que comúnmente se relacionan con esta dimensión son; entre otras, comparar, clasificar, hacer inducciones y deducciones.

Ejemplo: En un curso sobre zoología, apoyándose en artículos, videos, o animaciones en línea, presentar a los estudiantes las etapas evolutivas de los animales considerando peces, anfibios, reptiles, aves y mamíferos, caracterizando su sistema reproductor. Analizar las características del sistema reproductor y sus estrategias reproductivas y concluir, mediante un proceso de inducción, con las razones evolutivas de dicho sistema para cada uno de ellos. Las conclusiones deberán enviarse por correo electrónico para ser analizadas y publicadas en la sección de exhibición.

Dimensión 4: Usar el conocimiento significativamente, está relacionado, según los psicólogos cognoscitivistas, con el aprendizaje más efectivo, el cual ocurre cuando el educando es capaz de utilizar el conocimiento para realizar tareas significativas. Planear la instrucción para lograr esta dimensión es una de las decisiones más importantes que el profesor puede hacer. En este modelo instruccional se tienen cinco tipos de tareas que promueven el uso significativo del conocimiento; entre otros, la toma de decisiones, la investigación, la solución de problemas.

Ejemplo: En un curso sobre educación en línea, solicitar a los participantes que hagan una propuesta bien fundamentada (toma de decisiones) para instrumentar la modalidad de educación en línea en su institución, por lo cual deben analizar las características y requerimientos vía Internet de al menos tres de los programas de autoría y tres sistemas integrados que se puedan utilizar para instrumentar la modalidad. Esta propuesta debe ser enviada por correo-e para ser evaluada y publicada en la sección de exhibición.

Dimensión 5: Hábitos mentales productivos, sin lugar a dudas una de las metas más importante de la educación, se refiere a los hábitos que usan los pensadores críticos, creativos y con autocontrol, siendo los hábitos que permitirán el autoaprendizaje en el individuo en cualquier momento de su vida que lo requiera. Algunos de estos hábitos mentales incluyen; entre otros, ser claros y buscar claridad, ser de mente abierta, controlar la impulsividad, ser conciente de su propio pensamiento.

Ejemplo: En cualquier curso, hacia el final del mismo, solicitar a los alumnos que hagan un metaanálisis y reflexiones personales sobre las discusiones que se dieron en los Foros, incluyendo la cafetería virtual, este meta-análisis y reflexiones deben compartirse en el Foro de reflexión que se abrirá para ese fin.

\section{Consideraciones finales}

La propuesta presentada en este trabajo considera que el curso en línea será dado totalmente en línea, lo que implica que los participantes pudieran estar geográficamente distantes; sin embargo, no se debe descartar la posibilidad de instrumentarlo como apoyo a la práctica docente presencial. Concebirlo como apoyo a cursos tradicionales, habrá que adecuar algunas prácticas docentes que permitan explotar las bondades del medio y presentarlo no como una fuente optativa de información, sino como parte integral del currículo del curso. Del mismo modo, la estructura requerirá de algunas 
modificaciones en las secciones, por ejemplo, debido a la interacción cara a cara, el módulo de charla en tiempo real no sería de utilidad.

Esta propuesta debe ser evaluada en la práctica mediante la retroalimentación continua de docentes y estudiantes. Los docentes deben de evaluar la funcionalidad del curso y la respuesta y actitudes de los estudiantes además del desempeño académico de los mismos. Los estudiantes a su vez deben evaluar el ambiente de aprendizaje en su funcionalidad y estructura y al docente en cuanto a su papel como facilitador del aprendizaje y como instrumentador del modelo instruccional.

\section{Referencias}

MARZANO, R.J. A Different Kind of Classroom: Teaching with Dimensions of Learning. Alexandria, Va. USA: Association for Supervisión and Curriculum Development (ASCD). 1992.

MARZANO, R.J., D.J. Pickering, D.E. Arredondo, G.J. Blackburn, R.S. Brandt y C.A. Moffett. Dimensions of Learning Teacher's Manual. Alexandria, Va. USA: Association for Supervisión and Curriculum Development (ASCD). 1992.

MARZANO, R.J., D.J. Pickering y J. McTigthe (1993) Assessing Students Outcomes: Performance Assessment Using the Dimension of Learning Model. Alexandria, Va. USA: Association for Supervisión and Curriculum Development (ASCD). 1993.

McANALLY-SALAS, L.S. y C. Pérez-Fragoso, 2000. Diseño y evaluación de un curso en línea a nivel licenciatura. REDIE: Revista Electrónica de Investigación Educativa. Vol 2(1) Mayo del 2000. http://redie.ens.uabc.mx/

McISAAC, M.S. y Gunawardena, C.N. Distance Education. In: Jonassen, D.H. (ed) Handbook of research for educational communications and technology: a proyect of the Association for Educational Communications and Technology, New York, N.Y: Simon \& Schuster Macmillan, 1996. 